\title{
Perioperative Malignant Infarct in a Patient undergoing Mandibular Reconstruction with Free Fibular Flap Surgery for Oral Cancer: A Case Report
}

\author{
Kaminderbir Kaurl, Vishal Mangal ${ }^{2 *}$, Debashish Paul ${ }^{3}$, Kapil Kulkari ${ }^{4}$ \\ ${ }^{1}$ Clinical Tutor Department of Anaesthesiology and Critial Care, ${ }^{2}$ Clinical Tutor Department of \\ Internal Medicine, ${ }^{3}$ Associate Professor Department of Anaesthesiology and Critical Care, ${ }^{4}$ Junior \\ Resident Department of Anaesthesiology and Critical Care, Armed Forces Medical College, India
}

\begin{abstract}
Oral cancer is among the top three cancers in India. Surgery is the recommended treatment option for early-stage and locally advanced resectable cases. The incidence of perioperative stroke in patients undergoing neck dissection for head and neck cancer is only $0.2 \%$. In the following article, we report the first case of perioperative malignant infarct in a patient undergoing mandibular reconstruction with free fibula flap surgery for oral cancer. An eighty-year-old male diagnosed with locally advanced carcinoma of the oral cavity underwent mandibular reconstruction with free fibula flap surgery. In the perioperative period, he developed malignant infarction involving the right middle and posterior cerebral artery in the immediate postoperative period, however, it was diagnosed after 36 hours of surgery. He was managed conservatively and discharged with a favorable outcome. This case highlights the importance of clinical examination in such a case, along with the difficulties faced in examining the patient in the perioperative period.
\end{abstract}

Keywords: malignant infarct, perioperative, cancer, oral cavity, case report

\section{Introduction}

Oral cancer is among the top three types of cancers in India. ${ }^{1}$ The incidence of oral cancer increases with age, fifth decade being the commonest. $^{2}$ In particular, surgery and radiotherapy are the recommended treatment options for early-stage and locally advanced resectable cases. Organ preservation using systemic therapy is a less preferred choice. ${ }^{3}$ Perioperative stroke during head and neck surgery is a devastating complication with high morbidity and mortality. The incidence of perioperative stroke in patients undergoing neck dissection for head and neck cancer is only $0.2 \%{ }^{4}$ which is equal to the risk of perioperative stroke in patients undergoing non-head and neck

*Correspondence: Vishal Mangal

E mail: vishal rimshi@rediffmail.com

iD https://orcid.org/0000-0002- 7030- 5745

Received: $11 / 06 / 2020$

Accepted: 18/08/2020

DOI: http:/doi.org/10.4038/slja.v29i1.8611 surgery. The overall incidence of stroke for elderly patients (i.e., $\geq 65$ years) undergoing head and neck tumor surgeries, regardless of the extent of the operation, has been reported to be about $0.1 \% .^{5}$ The symptoms of stroke in the post-operative period are masked by immobilization of limbs, elective sedation, and inability to speak following resection of oral cavity tumor. Here we report a case of perioperative malignant infarct involving the right Middle and Posterior Cerebral Arteries most likely due to an embolus originating from the right subclavian artery due to handling during surgery for oral cavity cancer.

\section{Case Report}

An eighty-year-old male patient with no relevant past medical history was diagnosed with squamous cell cancer of the oral cavity following biopsy of an ulcer on the inner side of the right cheek of one-month duration. He was assigned, T4N2M0 staging after evaluation. He was planned for resection of the tumor and mandibular reconstruction with free flap from left fibula under general anaesthesia. Preoperative systemic examination and relevant laboratory investigations were within normal limits. The patient was classed as American 
Society of Anaesthesiologists class II. Airway examination did not reveal any apparent craniofacial deformity; the patient had adequate mouth opening with Mallampati class II and bilateral patent nares. On the day of surgery, after attaching the standard monitors, initial vital parameters were recorded, which were within normal limits. The induction was done with Injection Fentanyl 1microgram $/ \mathrm{kg}$ and Propofol up to $2 \mathrm{mg} / \mathrm{kg}$ in titrated doses. After confirmation of bag and mask ventilation, Injection Atracurium $0.5 \mathrm{mg} / \mathrm{kg}$ was administered. The patient was intubated nasally with an $8.0 \mathrm{~mm}$ internal diameter flexo-metallic endotracheal tube fixed at $26 \mathrm{~mm}$. Bilateral air entry was confirmed with auscultation. The surgery continued for eight hours, uneventfully. In view of prolonged surgery patient was kept intubated and sedated overnight with Injection Fentanyl 80 microgram/hour and midazolam $1 \mathrm{mg}$ /hour infusion.

The next morning sedation was discontinued, and the patient was put on pressure support ventilation. Subsequently, he was extubated over an airway exchange catheter. At the same time, it was observed that the patient was not moving his left lower limb (from where the fibula was harvested) and was not able to speak. This was attributed to tongue suture post-surgery, pain, and plaster in the left lower limb. However, the patient was fully conscious and following commands with adequate respiratory efforts. Thirty-six hours after the procedure, the patient developed tachycardia, a dip in sensorium with increased oxygen requirement on a facemask. On examination, he was drowsy; and was not moving his left lower limb at all with extensor response on planter reflex. The left upper limb also had a weakness with the power of grade $3 / 5$. The patient was taken for urgent noncontrast computed tomographic imaging of the head, which revealed an acute infarct (Figure 1a \& 1b).

The neurologist consultation was taken, and it was opined that the patient was not a candidate for thrombolytic therapy. He was managed conservatively with antiplatelets, lipid-lowering therapy, injection Mannitol 20\% $150 \mathrm{ml}$ intravenous infusion four times a day. The continuous oxygen requirement, impaired GCS causing a risk of aspiration and physically difficult airway warranted an elective
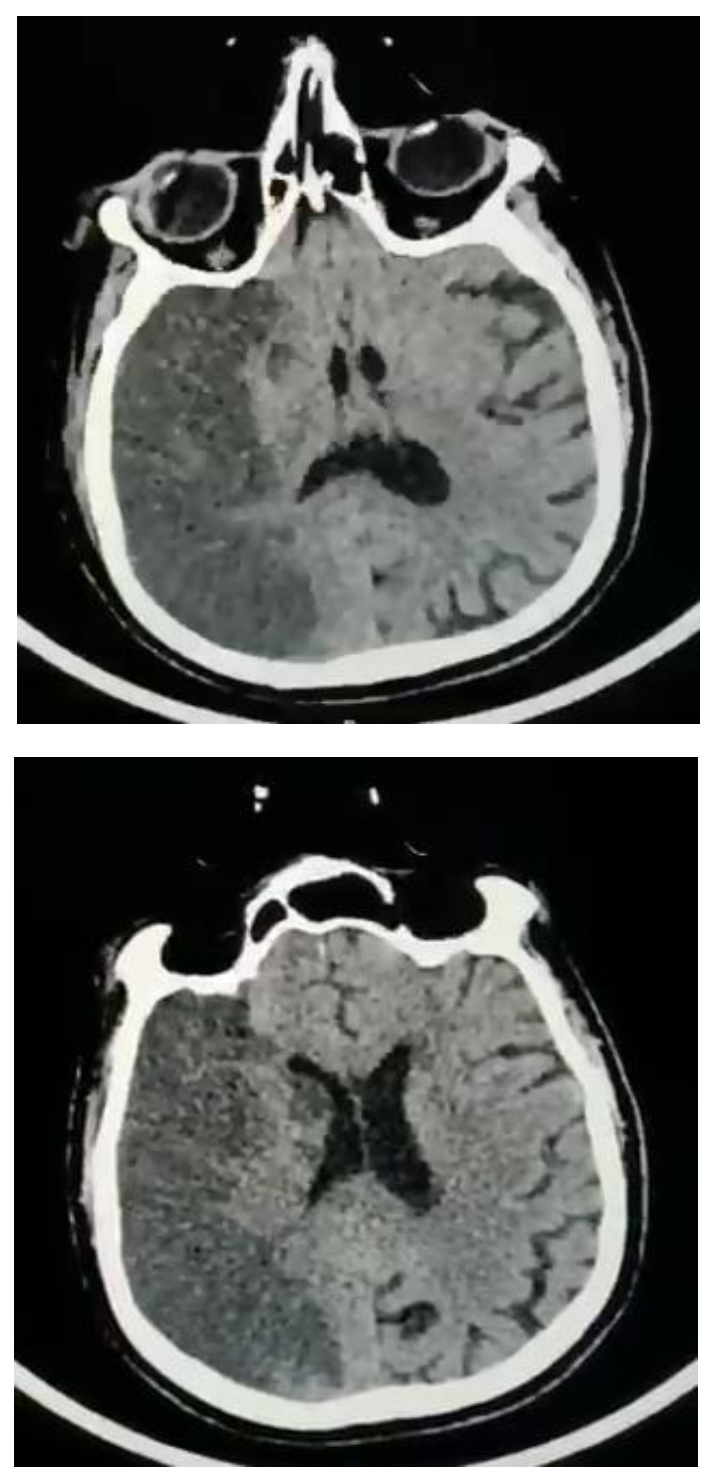

Figures 1a \& 1b

Non-contrast computed tomographic imaging of the head showing hypodensity in the right frontal, parietal and occipital lobes. There is associated cerebral edema with reduced grey white differentiation in the right fronto-parietotemporal lobes. There is mass effect in the form of effacement of the sulcal spaces, compression of the right lateral ventricle and third ventricle and a midline shift of three $\mathrm{mm}$ to the left.

tracheostomy, which was performed on the fourth postoperative day in ICU. The patient did not require ventilatory support but was put on Tpiece with an oxygen requirement of $4 \mathrm{~L} / \mathrm{min}$.

The patient recovered over a period of one week and was shifted to the ward without oxygen requirement; however, he was bedridden and required constant nursing care and attention. 
Subsequently, he was discharged after two weeks with tracheostomy tube in situ on room air. At the time of discharge, he was unable to walk without assistance and unable to perform activities of daily living without assistance. He scored four on the modified Rankin Scale.

\section{Discussion}

The reported risk of perioperative stroke is $0.1-$ $1.9 \%$ in non-cardiac, non-neurologic, and nonmajor surgery. ${ }^{6}$ Neck dissection is a recommended surgical procedure for head and neck cancer. $^{7}$ Neck dissection involves retraction of the neck vessels, causing dislodgement of a thrombus or plaque in patients with preexisting carotid artery atherosclerosis.

The term "malignant" was first used by Hacke et al. ${ }^{8}$ to characterize the complete infarction of the Middle cerebral artery (MCA) territory and is associated with about $80 \%$ mortality. The pathogenesis of ischemic stroke, in this case, was suspected to be dislodging of an embolus from the subclavian artery by neck dissection as the patient had involvement of both MCA and posterior cerebral artery territories.

As an institutional protocol, the fibula is chosen on the side other than that of the oral lesion so that both the onco-surgery and the reconstructive surgery team can start the procedure simultaneously. In our patient, a tongue suture was applied to prevent the tongue from falling back and causing airway obstruction. The left leg was placed in plaster following harvesting of the fibula. Most likely, the patient developed ischemic stroke in the immediate post-operative period, and it was not diagnosed as: 1) The patient was on continuous sedation for 12 hours following the surgery. 2) His left leg was in plaster as the fibula was harvested from this side. 3) The left upper limb power was reduced but not absent, and moreover, it had the catheter for invasive blood pressure monitoring. However, when the patient deteriorated after 36 hours of surgery, imaging revealed malignant infarct. This case highlights the difficulties faced by the clinicians in monitoring and examining these patients. Hence, a thorough neurological examination should be performed so that the subtle signs of neurological deficit can be diagnosed early. In this case all the typical symptoms of a stroke were not elicited because of either surgical or anesthetic interventions.
This is the first reported case in the literature of the successful management of the perioperative malignant infarct following Mandibular reconstruction and free fibula flap surgery for oral cavity cancer.

\section{Acknowledgements}

The authors are grateful to Dr Richa Joshi, Onco-surgeon for the successful management of the case.

\section{References}

1. Elango JK, Gangadharan P, Sumithra S, Kuriakose MA. Trends of head and neck cancers in urban and rural India. Asian Pacific Journal of Cancer Prevention. 2006; 7(1):108-12. PMID: 16629526

2. Rahman S, Sarker M, Khan MH, et al. Clinical profile of oral squamous cell carcinoma patients attending a tertiary care hospital. Bangladesh Medical Journal.2015;47(1-2):3-6

3. Chaturvedi P, Prabhash K, Babu G, et al. Indian clinical practice consensus guidelines for the management of oral cavity cancer. Indian Journal of Cancer 2020; 57(5): S6-8. https://doi.org/10.4103/0019-509x278975 PMID:32167064

4. Thompson S, Southern D, McKinnon J, et al. Incidence of Perioperative Stroke After Neck Dissection for Head and Neck Cancer. Annals of surgery. 2004; 239(3): 428-431 PMID:15075662

5. Mashour GA, Shanks AM, Kheterpal S. Perioperative stroke and associated mortality after non-cardiac, non-neurologic surgery. Anesthesiology. 2011; 114(6):1289-96. PMID: 21478735

6. Bateman BT, Schumacher HC, Wang S, et al. Perioperative acute ischemic stroke in noncardiac and nonvascular surgery: incidence, risk factors, andoutcomes. Anesthesiology. 2009; 110 (2):231-238

PMID:19194149

7. D'Cruz AK, Vaish R, Kapre N, et al. Elective versus therapeutic neck dissection in nodenegative oral cancer. The New England journal of medicine. 2015; 373(6): 521-529. https://doi.org/10.1056/NEJMoa1506007 PMID:_26027881

8. Hacke W, Schwab S, Horn M, et al. 'Malignant' middle cerebral artery territory infarction: clinical course and prognostic signs. Archives of neurology. 1996; 53(4): 309-315. https://doi.org/10.1001/archneur.1996.00550040 037012. PMID: 8929152 